\title{
Detection Rifampicin Resistance Tuberculosis Using Xpert MTB/RIF Assay in Saiful Anwar Hospital Malang
}

\author{
Dwi Yuni Nur Hidayati ${ }^{1}$, Yani Jane Sugiri ${ }^{2}$ \\ ${ }^{1}$ Microbiology Laboratory Medical Faculty Brawijaya University / Saiful Anwar Hospital \\ ${ }^{2}$ Pulmonary Departemen saiful Anwar Hospital \\ *e-mail: yuninur83@yahoo.com
}

\begin{abstract}
Tuberculosis is still problem in Indonesia. The increase number tuberculosis patientsand then multidrug resistance tuberculosis the higher it. Xpert MTB /RIF assay, an automated real time polymerase chain reaction assay, detects the presence Mycobacterium tuberculosis and rifampicin resistance to rifampicin in sputum within 2 hours. Here we report the epidemiology Mycobacterium tuberculosis of RIF - resistant TB pattern in Saiful Anwar Hospital Malang city. Material methods: A total of 3063 sputum specimen received in microbiology laboratory Saiful Anwar Hospital Malang between Januari 2013 until Desember 2016 were tested by Xpert MTB/RIF assay following manufactur's instruction.. Sputum specimen from patients suspect TB- MDR and TBHIV. Result : Total of 645 sputum specimen from TB- HIV patients suspect were detected rifampicin resistance $0,3 \%$ and $.21,1 \%$ rifampicin sensitive. The Total 2418 .from TB-MDR patients suspect were detected $8.1 \%$ rifampicin resistance and 46.6.\% rifampicin sensitive. Conclusions : Xpert MTB/RIF assay can use screening tool for detection of Mycobacterium tuberculosis and Rifampicin resistance from suspected TB-MDR and TB-HIV cases in a shorter periode of time.
\end{abstract}

Key word : Mycobacterium tuberculosis, Xpert MTB/RIF, resistance rifampicin

\section{INTRODUCTION}

Mycobacterium tuberculosis remains one of the most significant causes of death from an infectious agent. The incidence of HIV + TB in Indonesia is nearly 395 per 100,000 population, according to the 2015 Tuberculosis Reportin Indonesia (1). The proportion of multidrug-resistant (MDR) tuberculosis (TB) cases among new cases is $2.8 \%$. The rapid detection of M.tuberculosis and rifampin (RIF) resistance in infected patientsis essential for disease management, because of the high risk of transmission from person to person and emergenceof MDR-TB and extensively drug resistant tuberculosis. Culture is the "gold standard" for final determination, but it is slow and may take up to 2 to 8 weeks. Rapid identification, which is essentialfor earlier treatment initiation, improved patient outcomes, and more effective public health interventions, relies on nucleic acid amplification techniques. In Malang city the number of pulmonary tuberculosis patiens periods of 2016 increase to be 3,5\% from 1387 case in 2015 became 1437 case in 2016. Inability to rapidly diagnose and treat the affected patients leads to increased morbidity and mortality, development of secondary resistance (including extensively drug-resistant tuberculosis) and ongoing transmission of the disease (2). In this situation, not only rapid TB case detection, but also the early determination of MDR status is important. Conventionally the diagnosis of pulmonary tuberculosis has been based on clinical scenario, chest X-ray findings, smear microscopy for acid fast bacillus, or bacterial isolation by culture. In developing countries, out of all the lab investigations, diagnosis still relies heavily on the use of smear microscopy, which has a low sensitivity and specificity as compared to the culture. The microbiological identification of M.tuberculosis by culture remains the gold standard for diagnosis of tuberculosis. However, the conventional culture technique for 
Mycobacteria does not provide a rapid diagnosis, is a cumbersome procedure and requires sophisticated laboratory facilities of biological safety lab level II/III that cannot be afforded in most of resource limited settings (5). As an alternate, recent molecular diagnostic techniques are increasingly being promoted owing to their rapid turn around time and high sensitivity and specificity (5). The World Health Organization (WHO) has endorsed the implementation of GeneXpert MTB/RIF assay for national tuberculosis programsi $n$ developing countries (10). The Xpert MTB/RIF (Cepheid Inc.) is an automated, user friendly and rapid test based on nested realtime PCR assay and molecular beacon technology for MTB detection and RIF resistance. The results are obtained within a short period of 2 hours (5). Further on, the technique is not prone to cross-contamination, requires minimal Biosafety facilities and has a high sensitivity in smearnegative pulmonary TB. Here we report the epidemiology Mycobacterium tuberculosis of RIF resistant TB pattern in Saiful Anwar Hospital Malang city.

\section{METHODS}

A total of 3063 patients were received in microbiology laboratory of Saiful Anwar Hospital Malang City during a period of 2013 through 2016. The cohort was comprised of pulmonary tuberculosis $(n=2418)$. Multi Drug Resistant -Tuberculosis (MDR-TB) suspects and TBHIVsuspect ( $n=645$ ). Inclusion of 3063 pulmonary TB suspects was based on clinical symptoms (productive cough) for more than two weeks, persistent low-grade fever, night sweat and weight loss) and radiological findings consistent with tuberculosis. Sputum samples were collected from all these cases.

\section{Processing of samples:}

A. Sputum: All the sputum samples were subjected to:

1. Xpert MTB/RIF assay: Sputum samples were processed directly from Xpert MTB/RIF test, according to manufacturer's protocol. Sample reagent was added in a 2:1 ratio to unprocessed sputum in $15 \mathrm{ml}$ falcon tube and the tube was manually agitated twice during 15 minute incubation period at room temperature. Then $2 \mathrm{ml}$ of the inactivated material was transferred to the test cartridge by a sterile disposable pipette (provided with kits). Cartridges were loaded into the GeneXpert. The interpretation of data from MTB/RIF tests was software based and not user dependent (5).

\section{RESULTS}

Total of 645 sputum specimen from TB- HIV patients suspect were detected rifampicin resistance $0.3 \%$ and $21.1 \%$ rifampicin sensitive. The Total 2418 from TB-MDR patients suspect were detected $8.1 \%$ rifampicin resistance and $46.6 . \%$ rifampicin sensitive. 


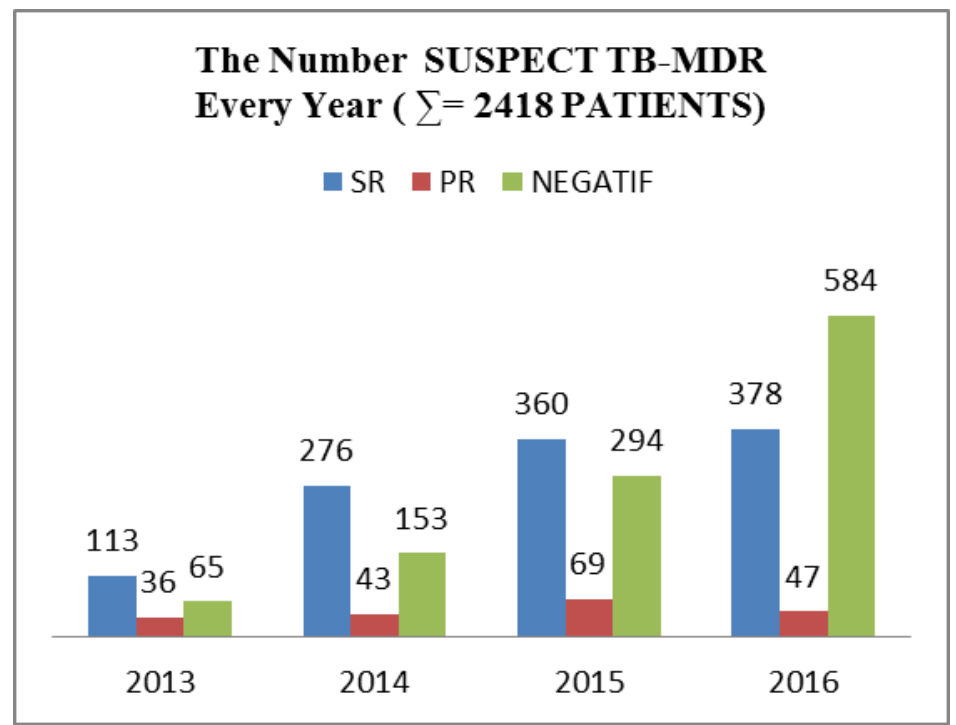

Figure 1. Overall results of Gene Xpert.Results TB-MDR Suspect

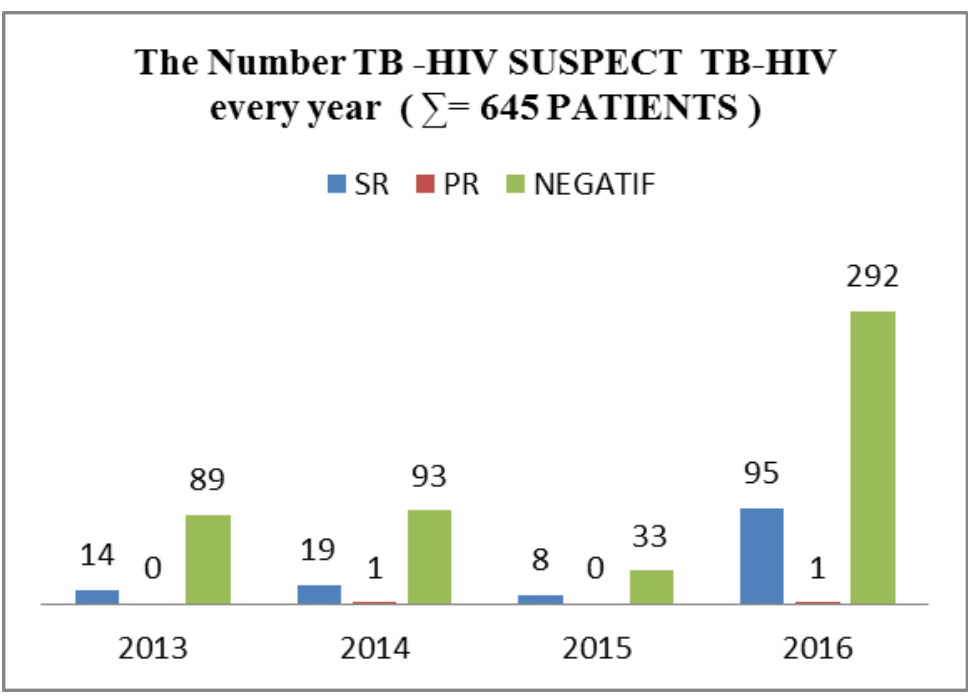

Figure 2. Overall results of Gene Xpert.Results TB-HIV Suspect

\section{DISCUSSION}

Conventional laboratory techniques as $\mathrm{ZN}$ smear microscopy for diagnosis of tuberculosis from clinical specimens is less sensitive as compared to the culture because large bacillary load $\left(10^{5} / \mathrm{ml}\right)$ will be required for a smear to become positive (8). Moreover the conventional cultures are time consuming and require Biosafety setup and trained laboratory personnels (9). The GeneXpertMTB/RIF assay is a rapid molecular biology/gene based assay that can be used close to the point of care by operators with minimal technical expertise. The technique enables diagnosis of Gene-Xpert for TB diagnosis and simultaneous assessment of rifampicin resistance to be completed within 2 hour (5). In the present study, we have evaluated the diagnostic accuracy of Xpert MTB/RIF assay for pulmonary cases and compared it with the conventional techniques. Here, the on time Xpert MTB/RIF assay could diagnose an additional $23.4 \%$ case along with an added advantage of much lower turn around time. Jahan, et al. in their study reported a better detection 
rate with Xpert when compared to smear microscopy and culture. This indicates that Gene Xpert is a highly sensitive technique for MTB detection compared to the conventional techniques.

\section{CONCLUSION}

Xpert MTB/RIF assay can use screening tool for detection of Mycobacterium tuberculosis and Rifampicin resistance from suspected TB-MDR and TB-HIV cases in a shorter periode of time. We suggest that in addition to its recommendeduse in MDR cases. We further recommend, more such studies shouldbe conducted to evaluate the feasibility of using thisinstrument in our local health care settings.

\section{REFERENCES}

1. World Health Organization. Global Tuberculosis Report 2015.

2. WHO (2011). Guidelines for the programmatic managementof drug-resistant tuberculosis. Geneva: World HealthOrganization.

3. Alvarez-Uria G, Azcona JM, Midde M, Naik PK, Reddy S,Reddy R. Rapid diagnosis of pulmonary and extrapulmonarytuberculosis in HIV-infected patients. Comparison ofLED fluorescent microscopy and the geneXpert MTB/RIFassay in a district hospital in India. Tuber Res Treatment.2012;2012:1-4.

4. Boehme CC, Nabeta P, Hillemann D, Nicol MP, Shenai S,Krapp F, et al. Rapid molecular detection of tuberculosisand rifampin resistance. N Eng J Med. 2010;363(11):1005-1015.

5. World Health Organization, Rapid Implementationof the Xpert MTB/RIF Diagnostic Test, World HealthOrganization, 2011.

6. Theron G, Peter J, van Zyl-Smit R, Mishra H, StreicherE, Murray S, et al. Evaluation of the Xpert MTB/RIFassay for the diagnosis of pulmonary tuberculosis ina high HIV prevalence setting. Am J Respir Crit CareMed. 2011;184(1):132-140.

7. Zeka AN, Tasbakan S, Cavusoglu C. Evaluation ofthe GeneXpert MTB/RIF assay for rapid diagnosisof tuberculosis and detection of rifampin resistancein pulmonary and extrapulmonary specimens. J ClinMicrobiol. 2011;49(12):4138-4141.

8. Lawn SD, Nicol MP. Xpert MTB /RIF assay : development, evaluation and implementation of New rapid moleculer diagnostic for tuberculosis and rifampicin resistance. Future Microbiol.2011;6(9):1067-1082

9. Moussa HS, Bayoumi F, Ali AM. Evaluation of GeneXpert MTB/RIF assay for direct diagnosis of pulmonary tuberculosis. Saudi Med J. 2016; 37(10):1076-1081

10. Jahan H, Jhora ST, Habib ZH, Yusuf MD, Ahmed I, Farzana A, Parveen R. Diagnostic Evaluation of Genexpert MTB/RIF Assay for Detection of Rifampicin Mycobacterium tuberculosis among Pulmonary Tuberculosis Patients in Bangladesh. Journal of Tuberculosis Research. 2016;4: 55-60 
11. Siu GKH, Zhang Y, Lau TCK et al. Mutation out side the rifampicin resistance - determinig region associated with rifampicin resistance in Mycobacterium tuberculosis. J Antimicrob Chemother. 2011; 66:730-733

12. Osman M, Simpson JA, Caldwell J, Bosman M, Nicol MP. GeneXpert MTB/RIF Version G4 for Identification of Rifampicin Resistant in Programatic Setting. Journal of Clinical Microbiology. 2014. 52(2) : 635-637 